\title{
Superconducting diamagnetic fluctuations in ropes of carbon nanotubes
}

\author{
M. Ferrier, ${ }^{1}$ F. Ladieu ${ }^{2}$ M. Ocio, ${ }^{2}$ B. Sacépé,${ }^{2}$ T. Vaugien,${ }^{3}$ V. Pichot,${ }^{1}$ P. Launois,${ }^{1}$ and H. Bouchiat ${ }^{1}$ \\ ${ }^{1}$ Laboratoire de Physique des Solides, associeé au CNRS UMR 8502, \\ Bâtiment 510, Université Paris-Sud, 91405 Orsay, France \\ ${ }^{2}$ SPEC CEA 91191 Gif/Yvette France \\ 3 Centre de Recherche Paul Pascal/CNRS UPR 8641, \\ Av. du Docteur Schweitzer, 33600 Pessac, France
}

\begin{abstract}
We report low-temperature magnetisation measurements on a large number of purified ropes of single wall carbon nanotubes. In spite of a large superparamagnetic contribution due to the small ferromagnetic catalytical particles still present in the sample, at low temperature $(T<0.5 K)$ and low magnetic field $(H<80 O e)$, a diamagnetic signal is detectable. This low temperature diamagnetism can be interpreted as the Meissner effect in ropes of carbon nanotubes which have previously been shown to exhibit superconductivity from transport measurements 1].
\end{abstract}

PACS numbers:

During the last decade, electronic properties of carbon nanotubes have attracted a lot of interest [5]. Depending on their diameter and helicity single wall carbon nanotubes (SWNT) are either semiconducting or metallic with in the latter case only 2 conducting channels at the Fermi energy. These long molecular wires constitute a model system for the investigation of one dimensional (1D) electronic transport and its great sensitivity to electron-electron interactions. The usual Fermi liquid is expected to be unstable in 1D, with the formation of a correlated Luttinger Liquid state [2]. In SWNT, interactions contain a strong repulsive Coulomb long range contribution, giving rise to a characteristic power law depression of the low energy tunneling density of states whose exponent is related to the interaction strength. Accordingly, non linear conductance measured on SWNT mounted on tunnel contacts has been interpreted as the signature of the existence of a Luttinger liquid state with repulsive interactions [3]. In this context the discovery of superconductivity below $0.5 \mathrm{~K}$ in transport measurements on SWNT ropes containing between 30 to 100 parallel $1.2 \mathrm{~nm}$ diameter tubes in good contact with normal electrodes [1, 4] was a surprise. This observation, which implies the existence of attractive interactions overcoming repulsive ones, has stimulated a number of theoretical investigations [6, 7, 8, 9, 10] of possible, in most cases phonon mediated, attractive mechanisms. In particular the famous acoustic breathing modes of SWNT 5 . have been shown to provide a phonon mediated attractive electron- electron coupling in SWNT when they are assembled into ropes. Repulsive Coulomb interactions are indeed strongly screened by the nearest neighbor metallic tubes, and there is also the possibility of an important inter-tube Josephson coupling [6, 9, 10]. A good agreement between theory and experiment was achieved concerning the broadening of the transition observed on the resistance versus temperature data when decreasing the number of tubes constituting the rope.

Beside transport, it is also essential to investigate ther-

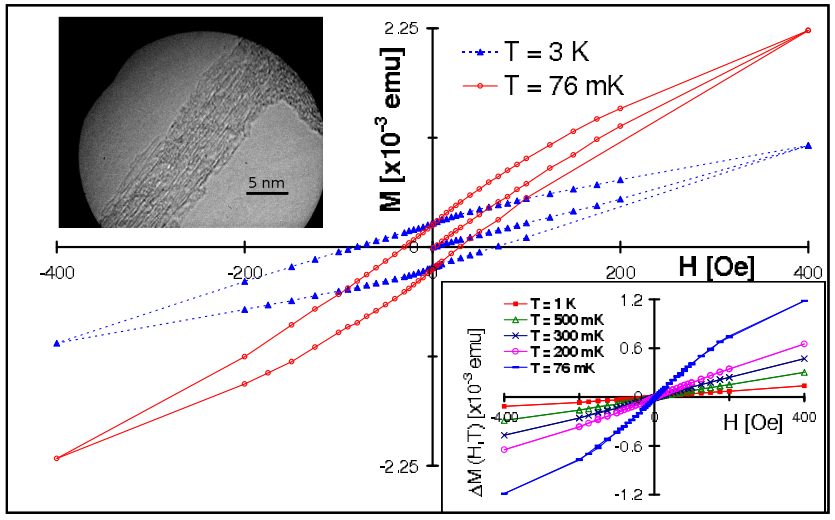

FIG. 1: Field dependence of the total magnetisation of the sample at various temperatures below $3.5 \mathrm{~K}$. Lower inset: field dependence of $\Delta M(T, H)=M(T, H)-M(3.5, H)$ for different temperatures showing that the hysteretic component of the magnetisation is independent of temperature below $3.5 \mathrm{~K}$. (This remains true up to $\mathrm{T}=35 \mathrm{~K}$.) Upper inset: transmission electron microscopy image of the SWNT ropes constituting the sample.

modynamic signatures such as the Meissner effect of this unusual superconductivity. The geometry of carbon nanotubes, which are very narrow $1 \mathrm{D}$ cylinders, is a priori not favorable for efficient magnetic flux expulsion. Nevertheless, magnetisation measurements performed on very small $(0.4 \mathrm{~nm})$ diameter SWNT, revealed a diamagnetic contribution increasing at low temperature below $6 \mathrm{~K}[11]$ which was interpreted as superconducting fluctuations. However the geometry of the samples grown in zeolite matrices was not adequate for transport measurements, which did not show clear sign of superconductivity.

The aim of the present work is to address this question of flux expulsion (i.e. Meissner effect) in ropes of SWNT similar to those previously studied in transport measurements [1, 4].

The samples consist of SWNT ropes (containing typically 10 to 100 parallel tubes whose diameter varies be- 
tween 0.9 to $1.4 \mathrm{~nm}$, the intertube distance being of the order of $0.3 \mathrm{~nm}$ (see fig 11). They were fabricated by decomposition of $\mathrm{Fe}(\mathrm{CO})_{5}$ according to the HiPCO process [12]. Removal of the Fe catalyst was achieved through annealing under Oxygen at $300{ }^{\circ} \mathrm{C}$ and subsequent treatment with hydrochloric acid. After these operations the amount of nanoparticles of Fe both in reduced or oxidized forms is estimated to be of the order of $1 \%$. These ropes were assembled into long ribbons in a PVA solution using the method described in [13]. The spreading of alignment of the nanotubes within a single ribbon was determined from Xray diffraction measurements according to the method described in [14] and found to be \pm 35 . For the purpose of this experiment these ribbons were wrapped around two 7 by $7 \mathrm{~mm}$ high purety saphyre substrates, which were subsequently annealed 3 hours at 320

${ }^{\circ} \mathrm{C}$ in order to eliminate the PVA surrounding the ropes. The mass of SWNT ropes deposited in this procedure, was measured to be $5 \mathrm{mg}$ and $6 \mathrm{mg}$ on the two saphyre substrate. The spreading of alignment of the nanotubes after wiring on the saphyre is probably not better than \pm 45 '. We will see that this nonetheless permits a determination of the anisotropy of the magnetisation of the nanotubes. Magnetisation measurements were performed on the 2 samples in a home built extraction SQUID magnetometer [15] equipped with a dilution refrigerator insert whose base temperature is $70 \mathrm{mK}$. The thermalisation of the sample was done with a small bundle of high purity copper wires attached to the saphyre plates inserted into a tube made from a capton foil. The contribution of the empty sample holder, capton, saphyre and copper bundle was shown to be below $310^{-8} \mathrm{emu} / \mathrm{Oe}$ in the whole range of temperature $(70 \mathrm{mK}, 300 \mathrm{~K})$ and magnetic field (3 to 3000 0e) investigated.

The magnetic signal contains a large ferromagnetic like hysteretic contribution. One sees on Fig 1 that this hysteresis does not change with temperature from $70 \mathrm{mK}$ up to $3.5 \mathrm{~K}$. This remains true up to $35 \mathrm{~K}$ (not shown). At any temperature below $35 \mathrm{~K}$, we thus decompose the magnetic signal into a $\mathrm{T}$ independent contribution attributed to frozen large magnetic particles and into a temperature dependent part which is reversible (i.e non hysteretic). In the following we mainly focus on this reversible low temperature dependent part of the magnetisation of the samples. We will show that beside the contribution of unfrozen superparamagnetic particles, it is possible to identify the presence of a diamagnetic contribution growing below $0.4 \mathrm{~K}$ which will be interpreted as the Meissner effect of the carbon nanotubes.

The magnetisation as function of temperature is plotted in fig.2-A. $M(T, H)$ increases monotonously as the temperature is lowered below $35 \mathrm{~K}$ down to $70 \mathrm{mK}$. This temperature dependent signal corresponds approximatively to $50 \%$ of the total magnetisation of the sample. The data was systematically taken in the field cooled state [16]. It is possible to superimpose all the curves

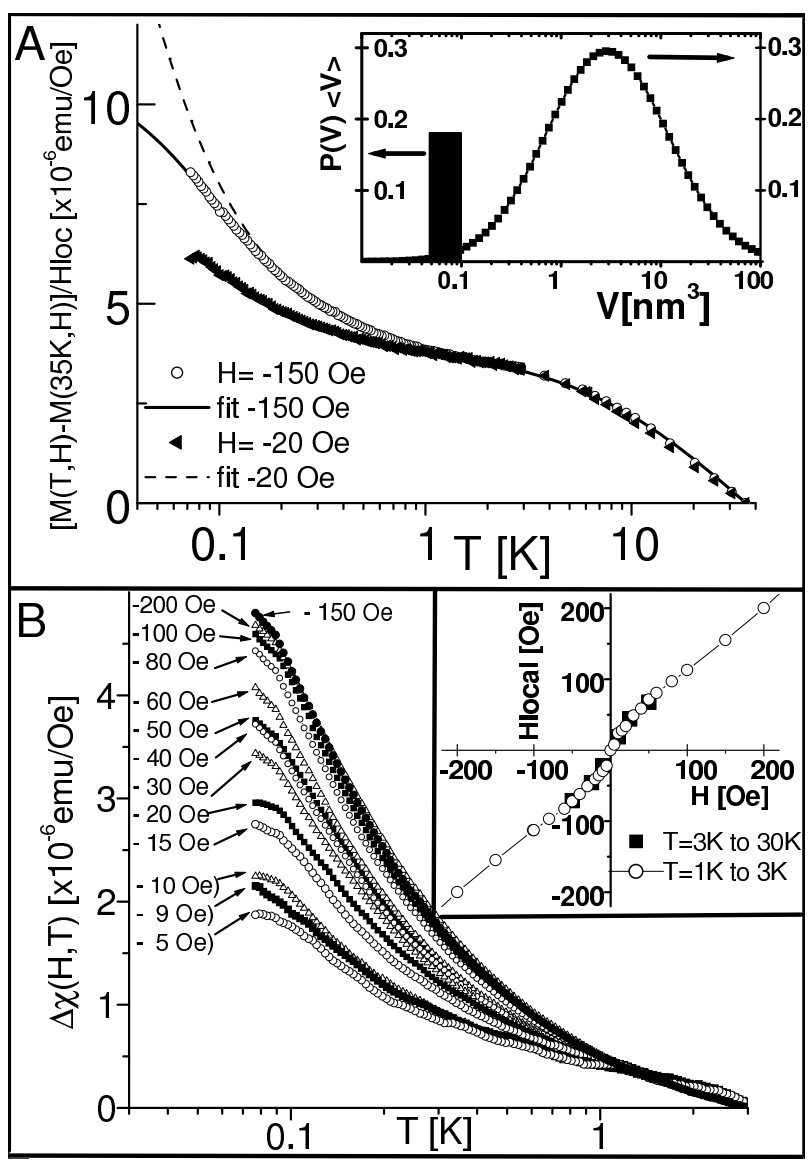

FIG. 2: (A)Renormalised magnetisation $M(T, H)-$ $M(35 K, H) / H_{l o c}(H)$ as a function of temperature between $70 \mathrm{mK}$ and $35 \mathrm{~K}$ for $\mathrm{H}=20$ Oe and $\mathrm{H}=150$ Oe compared to the fit with 2 assemblies of superparamagnetic particles whose adjusted volumic distributions are shown in the inset. Note the deviations at low temperature for the data taken at 20 Oe.(B)Temperature dependence of the field cooled renormalised magnetisation: $\Delta \chi(T, H)=\Delta M(T, H) / H_{l o c}(H)$ for various values of magnetic field. Inset: Field dependence of the local field obtained by renormalisation of the temperature dependent data above $1 \mathrm{~K}$ up to $3.5 \mathrm{~K}$ (circles) and up to $30 \mathrm{~K}$ (squares)

taken at various values of magnetic field below 150 Oe and above $1 \mathrm{~K}$ by renormalisation of the data with a field dependent factor $H_{\text {local }}(H)$ which corresponds to the effective average magnetic field inside the sample. As shown in fig.2-B $H_{\text {local }}(H)$ deviates from the applied magnetic field at low field values which suggests that the actual field inside the sample has a contribution coming from the largest particles saturating at low fields (typically lower than $30 \mathrm{Oe}$ ) which adds to the applied field. We have checked that the determination of $H_{\text {local }}(H)$ does not depend on the temperature range chosen between 1 and $35 \mathrm{~K}$ to renormalise the curves. More over in this temperature range, as shown on the inset of fig. $2 \mathrm{~A}$, the quantity $M\left(T, H_{\text {local }}(H)\right)$ can be very well described 


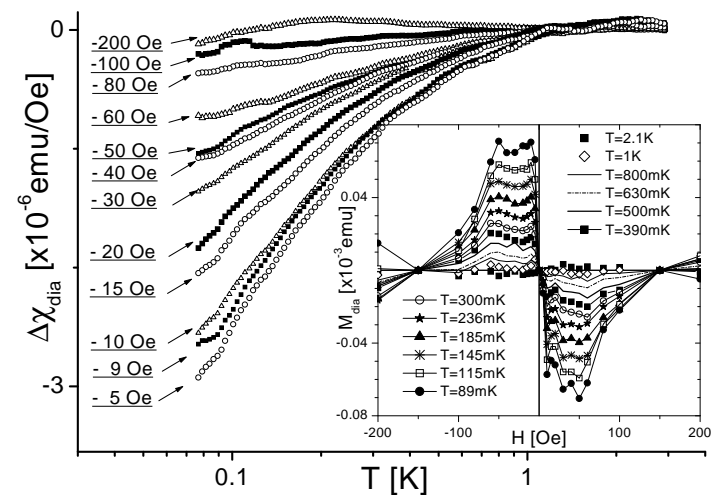

FIG. 3: Temperature dependence of the renormalised magnetisation after substraction of the data at $H=1500 e$ : $\Delta \chi_{\text {dia }}(T, H)=\Delta \chi(T, H)-\Delta \chi(T, 150)$.Inset: Reconstitution, using eq.4, of the field dependence of the diamagnetic contribution for various temperatures.

within a simple model of a volumic distribution of superparamagnetic $\mathrm{Fe}_{2} \mathrm{O}_{3}$ oxyde particles as inspired from previous work in ref. [17] where the anisotropy energy of $\mathrm{Fe}_{2} \mathrm{O}_{3}$ is taken to be $\mathrm{K}=5.410^{4} \mathrm{~J} / \mathrm{m}^{3}$. This distribution shown in the inset of fig.2-A, has a broad peak characteristic of a lognormal distribution around a particle volume which corresponds to typical diameters of the order of $1.5 \mathrm{~nm}$, most of these particles are frozen below $3-10 \mathrm{~K}$. To account of the very low $\mathrm{T}$ upturn of $\mathrm{M}(\mathrm{T})$ we have added to the previous contribution a Brillouin law with $\mathrm{J}=8$. This corresponds to very small $\mathrm{Fe}_{2} \mathrm{O}_{3}$ aggregates containing typically 3 or 4 coupled iron spins. The magnetic moments of these particles (whose anisotropy energy is estimated to be of the order of $20 \mathrm{mK}$ ) are unfrozen down to very low temperature. Finally, the amplitudes of these 2 superparamagnetic signals added to the ferromagnetic part (independent of temperature) corresponds to a number of typically $310^{17} \mathrm{Fe}$ atoms for a 5 mg sample i.e $1 \%$ of the sample. This is what is expected after purification, in agreement with previous magnetisation measurements made on similar samples [19] above $4 \mathrm{~K}$. On can see that the magnetisation signal at 150 Oe is very well described by this simple model between $60 \mathrm{~K}$ and $70 \mathrm{mK}[18]$. On the other hand important low T deviations from this model are observed for the data measured at 20 Oe, increasing below $0.5 \mathrm{~K}$.

If we now focus on this low temperature data below $0.5 \mathrm{~K}$, we can see on fig.2-B, that the temperature dependence of $\Delta \chi(T, H)=\Delta M(T, H) / H_{\text {local }}(H)$ with $\Delta M(T, H)=(M(T, H)-M(T=3.5 K))$ varies substantially with the magnetic field. Note that the magnetisation of an assembly of unfrozen super-paramagnetic particles of typical moment $\mu$ is indeed expected to exhibit at low temperature a non linear field dependence starting in the field range where $\mu H \sim k_{B} T$. This consideration can explain the data above 150 Oe quite well, where a decrease of the $\mathrm{T}$ dependence of $\Delta \chi$ with magnetic field is indeed observed (see the curve at 2000 e in fig.2). On the other hand the low field data is surprising. Below $1 \mathrm{~K} \Delta \chi$ is observed (see fig.2) to strongly decrease with decreasing magnetic field between 100 and 5 Oe. Such a behavior cannot correspond to any superparamagnetic system even in the presence of interactions between the particles leading to a spin-glass like state [20]. This effect, is emphasized in fig 3 showing the quantity $\Delta \chi_{\text {dia }}(T, H)$ after performing the subtraction:

$$
\Delta \chi_{\text {dia }}(T, H)=\Delta \chi(T, H)-\Delta \chi(T, 150)
$$

This plot strongly suggests the existence of a temperature dependent diamagnetic signal increasing rather smoothly at temperatures below $0.4 \mathrm{~K}$, for low magnetic field, superimposed to the superparamagnetic contribution of the catalyst particles. By subtracting data at 150 Oe instead of the superparamagnetic contribution inferred from the fit of fig.2-A, we probably underestimate this diamagnetic signal, on the other hand this method is model independent.

In the following we show that this diamagnetic contribution can be interpreted as screening diamagnetic supercurrents due to superconducting fluctuations in the ropes of carbon nanotubes. These ropes can be approximated by superconducting cylinders with diameter D small compared to the London penetration length $\lambda(T)$ (see for example the recent work on $\mathrm{Pb}$ superconducting nano-cylinders 21]). In the linear response regime,(observed in present case up to 10 Oe) the diamagnetic susceptibility (per unit volume) of a dense assembly of superconducting cylinders can be simply expressed as a function of $D / \lambda$ as:

$$
M_{\|, \perp}(H)=-H\left(D / \lambda_{\|, \perp}(T)\right)^{2}
$$

when the field is respectively parallel, perpendicular to the axis of the nanotubes within the rope. $\lambda_{\|}(T)$ and $\lambda_{\perp}(T)$ are respectively the penetration length parallel and perpendicular to the cylinders. Considering the anisotropy of the electronic structure of a single tube, as well as the existence of an inter-tube Josephson coupling, it is reasonable to assume that $\lambda_{\|}(T)$ and $\lambda_{\perp}(T)$ may be different from one another. In the absence of a more precise theoretical prediction, we use the simple London expression: $\lambda_{\|, \perp}^{-2}(T) \simeq 2 \mu_{0} n_{S}(T) e^{2} / m_{\perp, \|}$ where $n_{S}(T)$ is the density of superconducting pairs in the ropes and $m_{\perp, \|}$ are respectively the effective masses characterizing transport parallel and perpendicular to the ropes. Since only $1 / 3$ of the tubes within a rope are expected to be metallic, we assume that at zero temperature $n_{s}(0)=n_{e} / 6$, where $n_{e}=\frac{4}{a \pi d^{2} / 4}$ is the density of 
electrons in the 2 conduction bands of a metallic SWNT of diameter $d$ and $a$ is the graphene hexagonal lattice size. As a result, we get a simple expression relating the London diamagnetic susceptibility to the geometry of the carbon nanotubes ropes assumed to take random orientations through the sample (anisotropy effects will be discussed later).

$$
M_{a v}^{d i a}(H)=-H \frac{8 e^{2}}{3 m a \pi} \frac{D^{2}}{d^{2}}
$$

( $\mathrm{m}$ is approximated here by the free electron mass). From our measurements we can deduce the amplitude of the diamagnetic susceptibility in the linear regime to be $\chi_{\text {meas }}^{\text {dia }}=3 \pm 0.0510^{-6} \mathrm{emu} / \mathrm{Oe}\left(A m^{2} / T\right)$, reasonably close to the value given by expression (3) (multiplied by the volume of the sample), $\chi_{c a l}^{\text {dia }}=210^{-5} \mathrm{emu} / O e$ for an average diameter $D=10 \mathrm{~nm}$ per rope as estimated from transmission microscopy. This yields $\lambda(0) \simeq 0.6 \mu \mathrm{m}$ indeed much longer than the average diameter of the ropes. The temperature below which diamagnetism shows up $\left(T^{*}=0.4 K\right)$ is also very close to the values of transition temperature measured in transport measurements [1].

From the set of curves in fig [3] it is possible to reconstruct the field dependence of this diamagnetic component of the magnetisation for various temperatures, as shown in the inset:

$$
M_{\text {dia }}(H)=\Delta M(H)-H_{\text {local }}(H) \Delta \chi(150)
$$

$M_{\text {dia }}(H)$ measured at $70 \mathrm{mK}$ varies linearly at low field and goes through a maximum around 40 Oe, it decreases at higher field and becomes undetectable above 80 Oe. This behavior observed on both samples is typical of the magnetisation curves of a type 2 superconductor whose critical field $H_{c 1}$ is of the order of $60 \pm 20$ Oe. Note that this diamagnetic contribution at the lowest investigated values of magnetic field and temperature constitute more than $2 / 3$ of the temperature dependent magnetic signal below $3 \mathrm{~K}$ and $1 / 3$ of the total magnetic contribution.

We have also compared the amplitude of the magnetisation when the magnetic field is parallel to the directions of wrapping of the fibers compared to the situation where it is perpendicular. In both cases the field was maintained in the plane of the saphyre plate in order to avoid any macroscopic demagnetising factor. The paramagnetic component of the sample is found to be isotropic within an accuracy of $1 \%$. On the other hand it was possible to detect unambiguously on both samples an anisotropy of the Meissner component of the signal of the order of $10 \% \pm 1 \%$ indicating a larger amplitude of the Meissner component along the axis of the tubes than perpendicularly by a factor $1.2 \pm 0.05$ taking an angular distribution of the tubes axis of $\pm 45^{\circ}$ wide, (note that this number only slightly depends on the estimated spreading angle when its smaller than $60^{\circ}$ ). This result is however surprising since the Josephson coupling energy $t_{j}$ between the tubes is expected to be much smaller (at least by a facter 100) than the hopping integral $t$ along the tubes axis [6]. Nevertheless it is possible that the effective mass which characterize the intertube hopping of the Cooper pairs $m_{\perp} \propto 1 / d^{2} t_{j}$, is of the order and even smaller than along the tube axis $m_{\|} \propto 1 / a^{2} t$ since $d / a \simeq 10$.

All these findings corroborate the superconductivity observed in transport measurements on individual ropes of carbon nanotubes. Note that the diamagnetic part of the magnetisation disappears at relatively low magnetic field, more than a factor 10 lower than the critical field of the order of $1 \mathrm{~T}$ observed in transport experiments. This can be understood considering that the weak Josephson energy coupling between the tubes within one rope is destroyed at much weaker magnetic field than superconductivity within a single tube giving rise to a diamagnetic signal per unit volume of the order of $d^{2} / \lambda^{2}$ (undetectable in this experiment).

In all this analysis we have not taken into account the contribution of the orbital magnetism of the tubes in the normal state. Such orbital currents have been predicted and measured in multiwall nanotubes [22]. They are related to the sensitivity of electronic eigenstates and energies to the phase of the boundary conditions modified by the magnetic field. The resulting magnetic orbital susceptibility is diamagnetic for semiconducting tubes and paramagnetic for metallic tubes [23] and can be estimated to be at most $\chi_{\text {orb }}=210^{-8}$ emuOe $^{-1}$ for our sample, which is negligible compared to the diamagnetic contribution we have discussed. Our findings also present qualitative similarities with the results obtained by Tang et al. 11] on $0.4 \mathrm{~nm}$ diameter tubes, except that temperature and field scales are much smaller in our experiment. This could simply be related to the diameter of the tubes which are in the present case 3 times larger than in the Tang experiment. The characteristic energy of the phonon breathing mode, expected to be at the origin of superconductivity [8, [9] is known to scale as the inverse tube diameter. Moreover the possibility of the existence of a Wentzel Bardeen instability 24] specific to electron phonon coupling in a 1D system is predicted in very small diameter tubes, [8] favoring superconducting fluctuations against the formation of a Luttinger liquid with repulsive interactions, even in the situation where they are not well screened. Finally we note that the Kondo effect, leading to a dynamical screening of the magnetic moments at low temperature, could also give rise to a low temperature decrease of the magnetic susceptibility as observed in noble metals with a low concentration of magnetic impurities. To our knowledge there is no estimate of the order of magnitude of the Kondo temperature of iron magnetic impurities in carbon nanotubes. Since the electronic density of states is very small in carbon nanotubes compared to standard metals we expect this Kondo temperature to 
be also extremely small. Even if such a Kondo physics scenario cannot completely be ruled out to explain the depression of the Curie law at low field and low temperature it could not provide a description of the field dependence of the data shown in fig 3

In conclusion, we have shown that magnetisation of ropes of SWNT, at low temperature and magnetic field, cannot be explained by the superparamagnetism of the catalytical particles present in the sample. The weaker relative increase of magnetisation at small magnetic field compared to higher one, provides a strong indication of the existence of a diamagnetic Meissner contribution in ropes of SWNT increasing below $0.4 \mathrm{~K}$, in agreement with the onset of superconductivity observed in transport measurements realised on similar samples. From the alignment of the tubes in the samples, although imperfect, it was also possible to probe the anisotropy of the Meissner effect.

We thank S.Gueron, R.Deblock, M.Kociak, P.Poulin, S.Senoussi, L.Lepape, E.Vincent, J.Hamman and P.Bonville for fruitful discussions and help.

[1] M. Kociak, A. Kasumov, S. Guéron, B. Reulet, I. I. Khodos, Y. B. Gorbatov, V. T. Volkov, L. Vaccarini, and H. Bouchiat, Phys. Rev. Lett. 86(11), 2416 (2001).

[2] R. Egger and A. O. Gogolin, Phys. Rev. Lett. 79(25), 5082 (1997).

[3] M. Bockrath, D. Cobden, J. Liu, A. Rinzler, R. Smalley, L. Balents, and P. McEuen, Nature 397, 598 (1999).

[4] A. Kasumov, M. Kociak, M. Ferrier, R. Deblock, S. Guéron, B. Reulet, I. Khodos, O. Stéphan, and H. Bouchiat, Phys. Rev. B 68, 214521 (2003).

[5] M. Dresselhaus, G. Dresselhaus, and P. Eklund, Science of Fullerenes and Carbon Nanotubes, Academic Press, San Diego, CA, 1996.

[6] J. Gonzalez, Phys. Rev. Lett. 88(7), 76403 (2002).J. Gonzalez, Phys. Rev. B 67, 014528 (2003).
[7] A. Sédéki, L. G. Caron, and C. Bourbonnais, Phys. Rev. B 65, 140515 (2002).

[8] A. D. Martino and R. Egger, Phys. Rev. B 67, 235418 (2003).

[9] A. De Martino and R. Egger, Phys. Rev. B 70, 014508 (2004).

[10] M. Ferrier, A. de Martino, A. Kasumov, S. Guéron, M. Kociak, R. Egger, and H. Bouchiat, Solid. Stat. comm.131, 615 (2004).

[11] Z. Tang, L. Zhang, N. Wang, X. Zhang, G. Wen, G. Li, J. Wang, C. Chan, and P. Sheng, Science 292, 2462-2465 (2001).

[12] M. J. Bronikowski, P. A. Willis, D. T. Colbert, K. A. Smith, and R. E. Smalley, J. Vac. Sci. Technol. A 19,4 (2001).

[13] B. Vigolo, A. Pénicaud, C. Coulon, C. Sauder, R. Pailler, C. Journet, P. Bernier, and P. Poulin, Science 290, 1331 (2000).

[14] S. Badaire, V. Pichot, C. Zakri, P. Poulin, P. Launois, J. Vavro, C. Guthy, M. Chen and J. Fischer, J. Appl. Phys. 96, 7509-7513 (2004)

[15] R.Sappey, E.Vincent, M. Ocio, J.Hamman, F.Chaput, J.P.Boilot, D. Zins, Europhysics Letters, 37, 639, (1997).

[16] A small albeit systematic difference of the order of few $\%$ of $\Delta M(H, T)$ at $100 \mathrm{mK}$ could be noticed between the field cooled and zero field cooled data. We chose to focus on the FC data since it yields the under bound of $\left|M_{\text {dia }}\right|$.

[17] R. Sappey, E. Vincent, N. Hadacek, F. Chaput, J. P. Boilot, and D. Zins, Phys. Rev. B 56, 14551 (1997).

[18] Deviations to the fit observed at temperatures above $100 \mathrm{~K}$ can be attributed to the largest particles whose magnetisation freeze out in this temperature range contributing to the local field and not taken into account by the 2 distributions fit we have used.

[19] F. Chen et al. Appl. Phys. Lett., 83, 4602, (2003).

[20] In spin glasses at any given $\mathrm{T}, \mathrm{M}(\mathrm{H}) / \mathrm{H}$ is a decreasing function of $\mathrm{H}$.

[21] X.Y.Zhang and J.Y. Dai, Nanotechnology 15, 1166(2004)

[22] X. K. Wang, R. Chang, A. Patashinski, and J. Ketterson, J. Mater. Res. 9(6), 1578-1582 (1994).

[23] J. P. Lu, Phys. Rev. Lett. 74(7), 1123-1126 (1995).

[24] D. Loss and T. Martin, Phys. Rev. B 50, 12160 (1994). 